\title{
Metals Characterization by Principal Component EDS Analysis and EBSD
}

\author{
Keith Thompson ${ }^{1}$, Justin D. Morrow ${ }^{1}$ \\ 1. Thermo Fisher Scientific, Madison, WI, USA.
}

Electron Backscatter Diffraction (EBSD) is a technique for determining the near surface crystallographic and microstructural properties of a material. It is based on the automated capture and analysis of diffraction patterns formed when a sample is excited by an electron beam within the SEM. Energy Dispersive Spectroscopy (EDS) is a technique for determining the elemental composition of a material. The ED spectrometer collects the characteristic X-rays generated from the sample when it is similarly excited by an electron beam. When the ED spectrometer and the EBSD camera are aligned such that both instruments have a clear line of sight to the sample as it is excited by the electron beam, the material structure and chemistry can be determined simultaneously.

This simultaneous analysis is of particular value in metallurgy where the crystallographic structure and therefore the physical properties (e.g., strength and hardness) are influenced by local deviations from the nominal alloy chemical composition and the thermal and physical processes used in manufacturing. Both the primary manufacturing process (e.g., casting, forging) and secondary manufacturing processes (e.g., drawing, rolling, machining, welding) can impose thermal cycling and physical stress that will further influence the chemical composition and crystallographic structure. Simultaneously capturing both the microstructural phases and orientation (EBSD) and the elemental distribution (EDS) is key to understanding the impact of the starting alloy composition and subsequent manufacturing steps on the ultimate performance of any engineered product.

In this study, analyses were performed with a Thermo ScientificTM UltraDry EDS detector and Thermo Scientific Quasor EBSD. EDS maps were acquired at $15 \mathrm{kV}, 0.5 \mathrm{~mm}$ step and exposure of $1.5 \mathrm{~ms}$ per pixel. EDS phase mapping was performed using a principal component analysis (PCA) technique, as developed at Sandia National lab [1]. PCA is used to extract the dominant elemental components of the metal and to correlate those components to the underlying chemistry in each EBSD phase.

Figure 1 shows an example involving the analysis of a duplex steel, containing approximately equal amounts of $\alpha$-ferrite (BCC) and $\gamma$-Austenite (FCC) grains. Of particular interest is the grain size, distribution and orientation of both phases as well as how these phases are mixed in the microstructure as this gives insight into the ductility and strength of the material. The higher magnification image, Fig. 1c, shows the COMPASS phase maps from the EDS analysis. In this case, the $\alpha$-ferrite phase contains $4 \%$ additional chromium and $2 \%$ additional molybdenum while the $\gamma$-austenite contains $6 \%$ additional nickel. While subtle, this difference in elemental distribution is sufficient to locally change the most thermodynamically stable crystal phase and results in a dual-phase microstructure with useful engineering properties.

Figure 2 shows another example where simultaneous acquisition and analysis of diffraction patterns and characteristic x-rays provides insight into the combined microstructure and chemical composition of a quenched steel. The $\alpha$-ferrite (BCC) has smaller grains at the edges of the $\gamma$-austenite (FCC) grains. The BCC phase has $5 \%$ added $\mathrm{Cr}$ relative to the FCC phase, while the FCC phase has $2 \%$ added Ni. This implies that the material was uniformly $\gamma$-austenite (FCC) at elevated annealing temperatures and that as the metal cooled, a diffusion-mediated phase transformation occurred at the grain boundaries as the $\mathrm{Cr}$ was rejected from the grains during cooling. The higher Ni content is associated with an FCC structure while elevated $\mathrm{Cr}$ leads to $\mathrm{BCC}$ grains. An interesting banding pattern can also be seen in the images 
indicating that this was a non-equilibrium process and may have been affected by pre-existing texture in the starting $\gamma$-austenite (FCC) grains and a short cooling time that limited time for $\mathrm{Cr}$ diffusion. These insights into the likely heat-treatment cycle of the material would not be possible without confidence in both the chemical composition and crystallographic structure.

References:

[1] PG Kotula and MR Keenan, Microsc Microanal 12 (2006), p. 538.

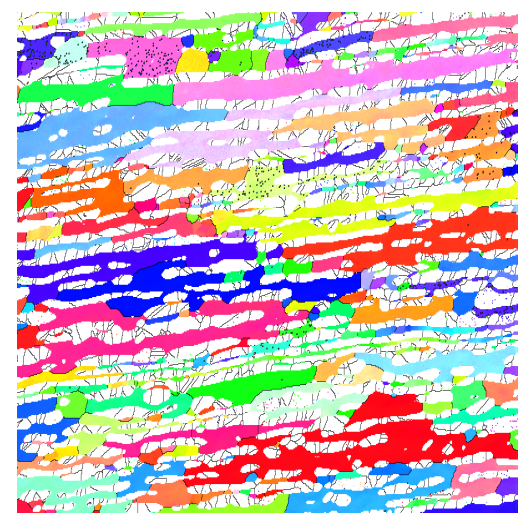

Figure 1. Concurrent EDS and EBSD analysis of a duplex steel: a) orientation distribution of a-phase ferrite, b) orientation distribution of g-phase austenite, c) High magnification EDS phase maps for a-phase Ferrite (yellow) and g-phase Austenite (red) within the Duplex, d) elemental composition of each phase by weight percent.

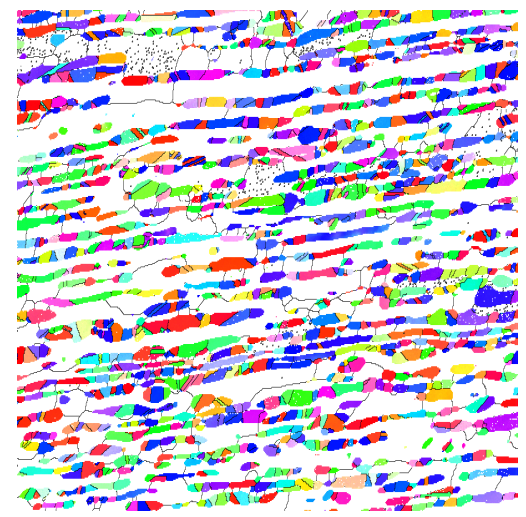

\begin{tabular}{|ccc} 
Element & $\begin{array}{c}\text { Austenite } \\
\text { Weight \% }\end{array}$ & $\begin{array}{l}\text { Ferrite } \\
\text { Weight \% }\end{array}$ \\
\hline Cr & 21.44 & 25.10 \\
Mn & 3.36 & 3.35 \\
Fe & 60.89 & 61.29 \\
Ni & 10.09 & 4.15 \\
Mo & 4.22 & 6.11 \\
Total & 100.00 & 100.00 \\
\hline
\end{tabular}

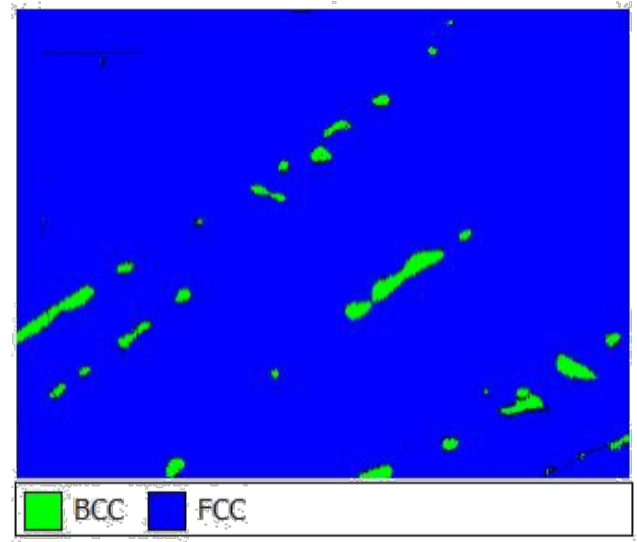

Crystal phases

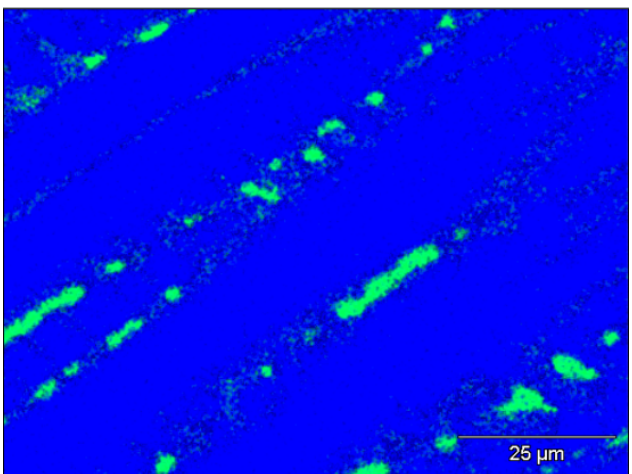

Elemental phases

Figure 2. Concurrent EDS and EBSD analysis of a quenched stainless steel sample: (left) crystal phases as determined by EBSD; (center) Elemental phases as determined by EDS using PCA; (right top) d.) corresponding EDS spectra for each phase; (right bottom) bar chart showing quantitative results for each element in each phase.
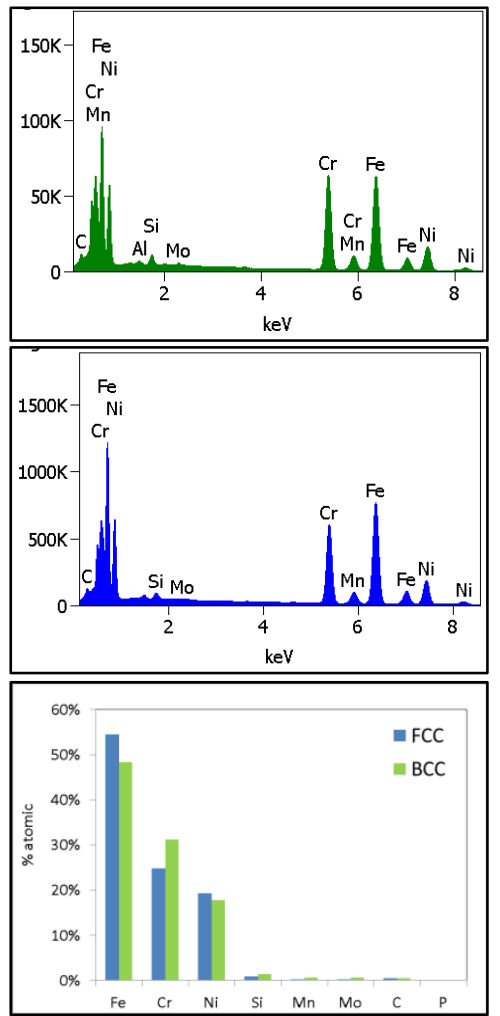